\title{
IN SILICO STUDY OF APIGENIN AND APIGETRIN AS INHIBITOR OF 3-HYDROXY-3-METHYL-GLUTAYL-COENZYME A REDUCTASE
}

\author{
DWINTHA LESTARI ${ }^{1 *}$, ELIN YULINAH SUKANDAR ${ }^{1}$, IRDA FIDRIANNY² $^{2}$
}

${ }^{1}$ Department of Pharmacology-Clinical Pharmacy, School of Pharmacy, Bandung Institute of Technology, Bandung, Indonesia. ${ }^{2}$ Department of Pharmaceutical Biology, School of Pharmacy, Bandung Institute of Technology, Bandung, Indonesia. Email: dwintha_85@yahoo.co.id

Received: 05 June 2017, Revised and Accepted: 31 July 2017

\section{ABSTRACT}

Objective: The objectives of this research were to investigate in silico interaction between apigenin and apigetrin with 3-hydroxy-3-methyl-glutaylcoenzyme A (HMG Co-A) reductase, to find the most favorable binding site as well as to predict the binding mode.

Materials and Methods: Docking calculation was performed by branded Sony Vaio PC Linux Ubuntu 14.04 LTS. The binding process based on the best docking result with HMG Co-A reductase was presented in two-dimensional diagram. Statin, atorvastatin, and R-mevalonate were used as standard.

Results: Binding affinity and inhibition constant of R-mevalonate were Ei=-4.2 kcal/mol, Ki=836.78 $\mu \mathrm{M}$; apigenin Ei=-7.0 kcal/mol, Ki=7.43 $\mu \mathrm{M}$; apigetrin Ei=-5.9 kcal/mol, Ki=47.53 $\mu \mathrm{M}$; simvastatin $\mathrm{Ei}=-8.2 \mathrm{kcal} / \mathrm{mol} ; \mathrm{Ki}=0.98 \mu \mathrm{M}$; atorvastatin Ei=-8.4 kcal/mol; $\mathrm{Ki}=0.7 \mu \mathrm{M}$. Apigenin had better binding interaction than apigetrin.

Conclusion: Apigenin could be developed as anticholesterol.

Keywords: Anticholesterol, In silico, Apigenin, Apigetrin, 3-hydroxy-3-methyl-glutayl-coenzyme A reductase.

(C) 2017 The Authors. Published by Innovare Academic Sciences Pvt Ltd. This is an open access article under the CC BY license (http://creativecommons. org/licenses/by/4. 0/) DOI: http://dx.doi.org/10.22159/ajpcr.2017.v10i11.20493

\section{INTRODUCTION}

There are four major types of lipoproteins, classified by their densities as measured in the ultracentrifuge: (1) Very low density lipoproteins, which contain high concentrations of triglycerides and moderate concentrations of both cholesterol and phospholipids; (2) intermediatedensity lipoproteins, which are very low-density lipoproteins from which a share of the triglycerides has been removed, so that the concentrations of cholesterol and phospholipids are increased; (3) lowdensity lipoproteins, which are derived from intermediate-density lipoproteins by removing almost all of the triglycerides, leaving an, especially high concentration of cholesterol and a moderately high concentration of phospholipids; and (4) high-density lipoproteins, which contain a high concentration of protein (about 50\%) but much smaller concentrations of cholesterol and phospholipids [1]

Apigenin $\left(4^{\prime}, 5,7\right.$-trihydroxyflavone $)$ is a natural product belonging to the flavone class that is the aglycone of several naturally occurring glycosides. In in vitro experiments and animal studies, various biological activities of apigenin have been reported. Apigenin may support a possible chemopreventive role by inducing autophagy (a kind of cellular waste-recycling system) in leukemia cells. Inducing in autophagy will interfere action of chemotherapy drug vincristine [2]. Apigenin was used for stem cell [3] and a potent inhibitor of CYP2C9 [4] an enzyme responsible for the metabolism of many pharmaceutical drugs in the body. Apigenin demonstrated to prevent renal damage which was caused by cyclosporine in rats, associated with reducing in expression of the cell death mediator bcl-2 in histopathological sections [5]. Cyclosporine enhances the expression of transforming growth factor- $\beta$ in the rat kidney, which signifies accelerated apoptosis. Therefore, transforming growth factor- $\beta$ and apoptotic index may be used to evaluate apigenin and its effect on cyclosporine-induced renal damage [6]. In vitro previous research revealed that apigenin may be toxic to red blood cells [7]. Several in vitro and in vivo studies presented anti-inflammatory, antidiabetic, and antiobesity effects of apigenin. However, the long-term supplementary effects of low-dose apigenin on obesity were unclear; therefore, the protective effects of apigenin against obesity and related metabolic disturbances by exploring the metabolic and transcriptional responses in high-fat diet-induced obese mice were evaluated [8]. However, studies of other active compounds and molecular action mechanism were not explained.

Apigetrin, also known as apigenin-7-0-glucoside is a natural flavonoid glucoside. There are very few reports regarding its anticancer activity. In previous in silico research represented that Apigetrin can act as a multitargeted agent that targets six different anticancer drug target such as mouse double minute 2, phosphatidylinositol 3 kinase, focal adhesion kinase, topoisomerase-ii, dihydrofolate reductase, and P-glycoprotein. Apigetrin gave more negative-binding free energies as an indication of favorable interaction against these targets [9].

3-hydroxy-3-methyl-glutayl-coenzyme A (HMG Co-A) reductase is the rate-controlling enzyme of the mevalonate pathway, the metabolic pathway that produces cholesterol and other isoprenoids. In humans, the gene for HMG Co-A reductase is located on the long arm of the fifth chromosome [10]. HMG Co-A reductase is anchored in the membrane of the endoplasmic reticulum, and was long regarded as having seven transmembrane domains, which the active site located in a long carboxyl terminal domain in the cytosol. Evidence of more studies denoted that it contained eight transmembrane domains [11].

A drug with goog solubility properties will show better absorption and bioavailability. Almost $40 \%$ of the drug in the market presents low solubility in water. It is causing the drug slowly to be absorbed [12]. Statin, the 3-hydroxy-3-methylglutaryl-coenzyme A (HMG CoA) reductase are the most effective inhibitor for lowering cholesterol which catalyse conversion of HMG-CoA to mevalonate [13]. Simvastatin was developed by Merck and came into medical use in 1992 [14], and the most important medications needed in a basic health system [15]. Atorvastatin is a member of the drug class known as statins, which are used primarily as a lipid lowering agent and for preventing a correlation with cardiovascular disease. 


\section{MATERIALS AND METHODS}

\section{Material}

Docking calculation was performed by branded Sony Vaio PC Linux Ubuntu 14.04 LTS as the operating system, with Intel $2.40 \mathrm{GHzCore} \mathrm{i5,}$ chipset: Intel HM55, memory: 4 GB DDR3 SODIMM PC-8500, video: ATI Mobility Rade on HD 5430512 MB, hard drive: 500 GB Serial ATA 7200 RPM. The softwares used for docking preparation were Chem Bio Office Ultra 13.0 Suite (Trial Version), ligand Explorer Viewer 4.1.0 (Online: http://www.pdb.org/pdb/explore/), Q-SiteFinder (online: http:// www.modelling.leeds.ac.uk/qsitefinder/), YASARA 13.9.8, Autodock Vina, PyRx 0.8, MGLTools1.5.6 (Auto Dock Tools-1.5.6), Chimera 1.8. Virtual analysis of docking site was used Autodock Tools 1.5.6 and Pose View to generated two-dimensional (2D) diagrams of docking poses.

\section{Experimental}

The subjected receptor in this study was HMG Co-A reductase (Fig. 1). The structure of the receptor was obtained in pdb files from the protein data bank (www.pdb.org). The receptor code for HMG Co-A reductase was 4I6Y. Each receptor had been docked with pepstatin as native ligand, which has been known as aspartic protease inhibitor.

In preparation step, separation of native ligand for each receptor was carried out using Structure Protonation and Recognition System (SPORES) software. SPORES was employed to split the pdb file to protein and ligands using the splitpdb module. The protein was recognized, protonated, and stored as protein.mol2, while the reference ligand was also recognized, protonated, and stored as ligand_ref.mol2. The binding site searched automatically using bind module of PLANTS 1.2 with radius distance of $5 \AA$ from the coordinates of the location where the native ligand was located in the reference crystal structure. Python script from Autodock tools together with Open Babel 2.3.2 was also used to prepare the receptor and ligand to be docked using Autodock Vina by changing mol2 to pdbqt file extension. Validation of docking protocols was carried out by redocking native ligand to receptor. The root mean square

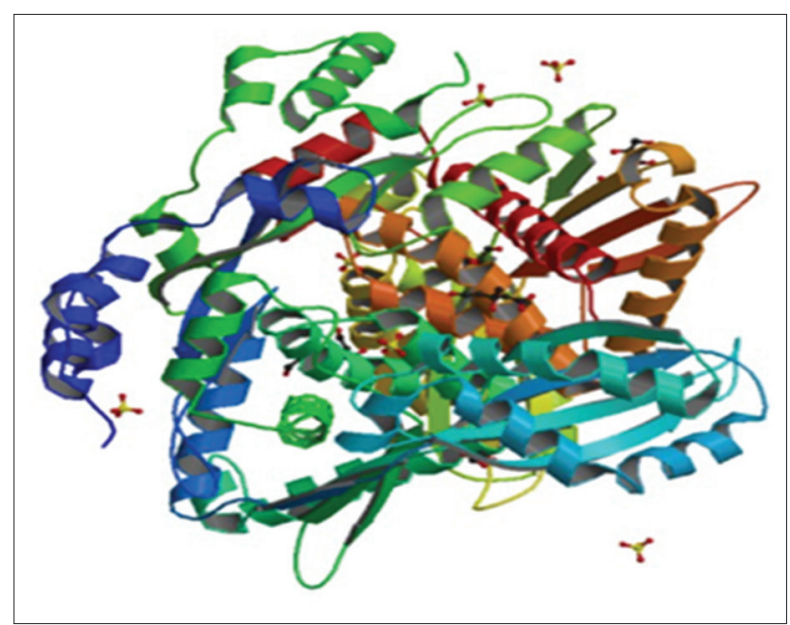

Fig. 1: Crystal structure of 3-hydroxy-3-methyl-glutayl-coenzyme A reductase deviation (RMSD) values calculations were performed using rms_cur module in PyMol. This process was repeated 100 times for each protein pdb files. The docking process was performed to HMG Co-A reductase as receptor, while simvastatin and atorvastatin, apigenin and apigetrin were used as ligands (Fig. 2). This process was iterated 100 times to each protein.

\section{RESULTS AND DISCUSSION}

Apigenin (5,7-dihydroxy-2-(4-hydroxyphenyl)-4H- chromen-4-one) is a naturally occurring, non-toxic, non-mutagenic phytonutrient flavonoid, commonly present in various fruits, and vegetables. Apigenin has long been considered to have various biological activities such as anti-inflammatory [16] and antitumorigenic effect [17] in various cell types. The basic structure of cholesterol is a sterol nucleus. In humans, cholesterol is derived from two sources - diet and de novo synthesis. All nucleated cells can synthesize cholesterol de novo from acetyl CoA through the mevalonate 12 pathway, which occurs in the ER. The rate limiting step in the mevalonate pathway is the conversion of HMG Co-A to mevalonate by HMG Co-A reductase. HMG Co-A reductase is an integral membrane protein which is under regulation through a negative feedback system modulated by the SREBP pathway [18]. The internal validation was aimed to examine whether the docking protocols can reproduce the pose of the cocrystal ligand [19]. The objective function used in the internal validation was the RMSD value between the heavy atoms of the docked pose and the crystal structure pose. The protocol is acceptable if the RMSD value is $<2.0$ [20]. By dividing the result of RMSD values from redocking process for each crystal structures (Table 1), it can be concluded that the docking protocols were valid.

The molecular docking results of reference ligand and test compounds were presented in Table 2 . The higher binding affinity demonstrated the better binding interaction with HMG Co-A reductase [21]. Atorvastatin showed better binding interactions with HMG Co-A reductase which gave higher binding affinity $(\mathrm{Ei}=-8.4 \mathrm{kcal} / \mathrm{mol})$ than others. Simvastatin and apigenin also presented good binding interaction, with binding affinity $\mathrm{Ei}=-8.2 \mathrm{kcal} / \mathrm{mol}$ and $\mathrm{Ei}=-7.0 \mathrm{kcal} / \mathrm{mol}$, respectively, while apigetrin showed the lowest binding affinity $(\mathrm{Ei}=-5.9 \mathrm{kcal} / \mathrm{mol})$ (Table 2 and Fig. 3).

The binding process of apigenin based on the best docking result with HMG Co-A reductase was presented in 2D diagram (Fig. 4).

Hydrogen bonds were formed with side chain of Gly281 (Fig. 4) whereas hydrophobic interaction with side chain.

Table 1: RMSD value of apigenin, apigetrin, simvastatin, and atorvastatin

\begin{tabular}{lllll}
\hline Ligand & $\begin{array}{l}\text { Binding afinity } \\
\text { (kcal/mol) }\end{array}$ & Mode & $\begin{array}{l}\text { RMSD } \\
\text { lower } \\
\text { bound }\end{array}$ & $\begin{array}{l}\text { RMSD } \\
\text { upper } \\
\text { bound }\end{array}$ \\
\hline 416Y_A_apigenin & -7.0 & 0 & 0.0 & 0.0 \\
416Y_A_apigetrin & -5.9 & 6 & 1.715 & 3.173 \\
416Y_A_simvastatin & -8.2 & 0 & 0 & 0 \\
416Y_A_atorvastatin & -8.4 & 0 & 0 & 0 \\
\hline
\end{tabular}

RMSD: Root mean square deviation

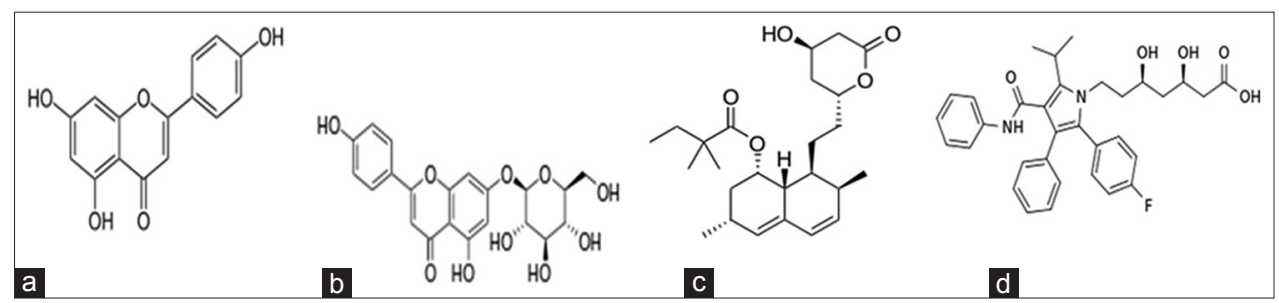

Fig. 2: Structure of (a) apigenin, (b) apigetrin, (c) simvastatin, (d) atorvastatin 
Table 2: Binding affinity to HMG Co-A reductase

\begin{tabular}{|c|c|c|c|c|c|c|c|c|c|c|}
\hline \multirow[t]{2}{*}{ Receptor } & \multicolumn{2}{|c|}{ R-mevalonate } & \multicolumn{2}{|c|}{ Apigenin } & \multicolumn{2}{|c|}{ Apigetrin } & \multicolumn{2}{|c|}{ Simvastatin } & \multicolumn{2}{|c|}{ Atorvastatin } \\
\hline & Ei & $\mathbf{K i}$ & Ei & $\mathbf{K i}$ & Ei & $\mathbf{K i}$ & Ei & $\mathbf{K i}$ & Ei & $\mathbf{K i}$ \\
\hline HMG Co-A reductase & -4.2 & 836.78 & -7.0 & 7.43 & -5.9 & 47.53 & -8.2 & 0.98 & -8.4 & 0.70 \\
\hline
\end{tabular}

Ei: Binding affinity (kcal/mol), Ki: Inhibition constant ( $\mu \mathrm{M})$, HMG Co-A: 3-hydroxy-3-methyl-glutayl-coenzyme A

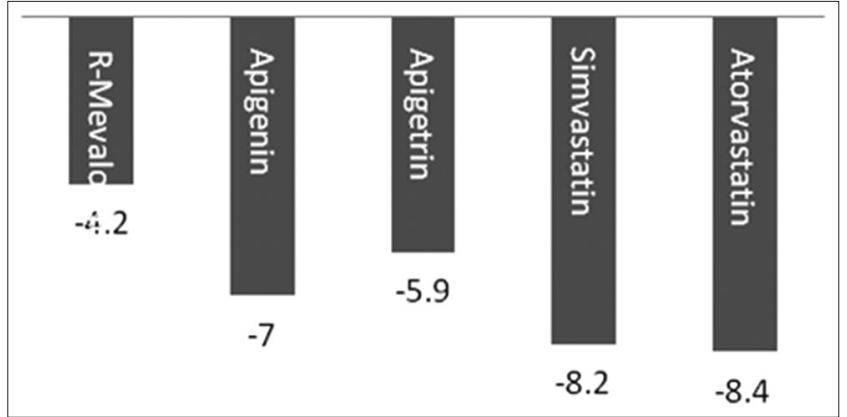

Fig. 3: Binding affinity to 3-hydroxy-3-methyl-glutayl-coenzyme A reductase

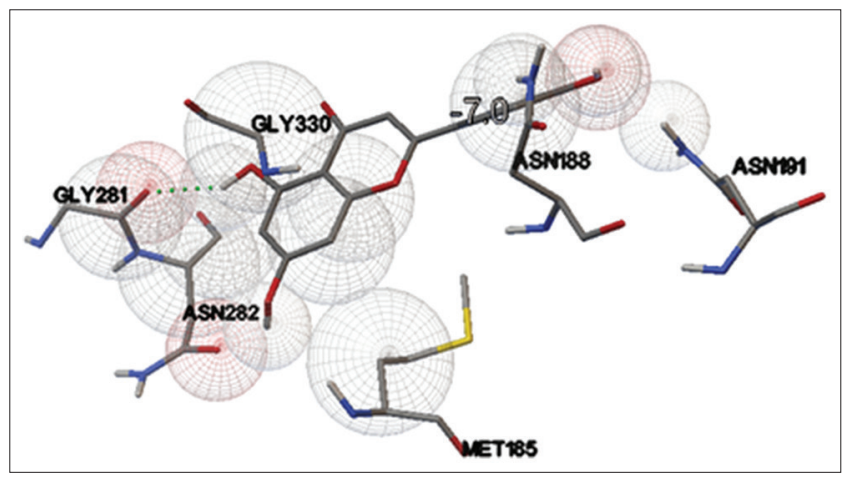

Fig. 4: Interaction of apigenin in the binding pocket of 3-hydroxy3-methyl-glutayl-coenzyme A reductase

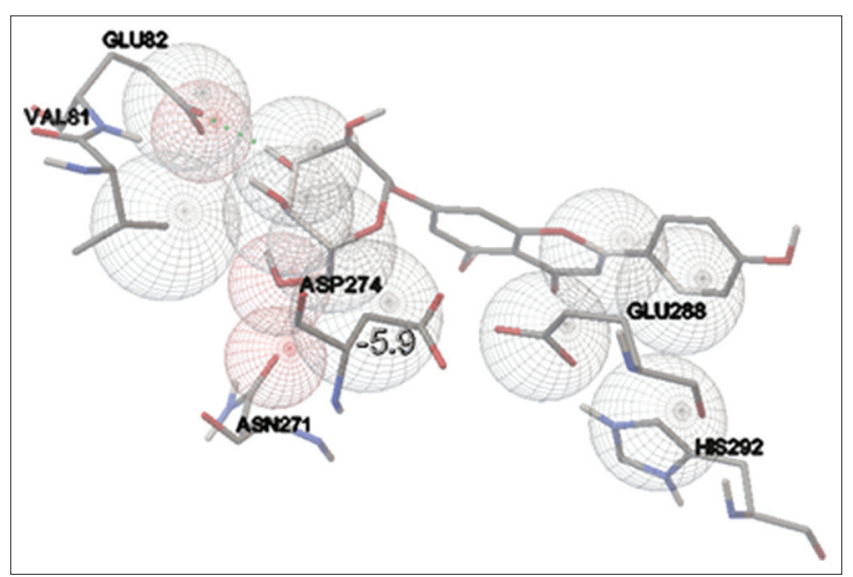

Fig. 5: Interaction of apigetrin in the binding pocket of 3-hydroxy3-methyl-glutayl-coenzyme A reductase

Interaction of apigetrin in the binding pocket of HMG Co-A reductase was exposed in 2D diagram, and hydrogen bonds was formed with side chain of Glu82 (Fig. 5).

The binding process of simvastatin and atorvastatin based on the best docking result with HMG Co-A reductase were presented in 2D diagram and hydrogen bonds were formed with side chain of SER66 (Fig. 6) and LEU60 (Fig. 7), respectively.

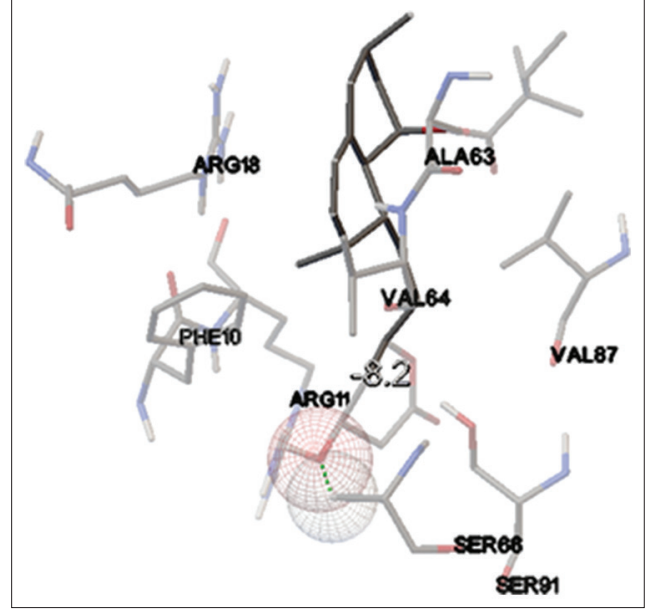

Fig. 6: Interaction of simvastatin in the binding pocket of 3-hydroxy-3-methyl-glutayl-coenzyme A reductase

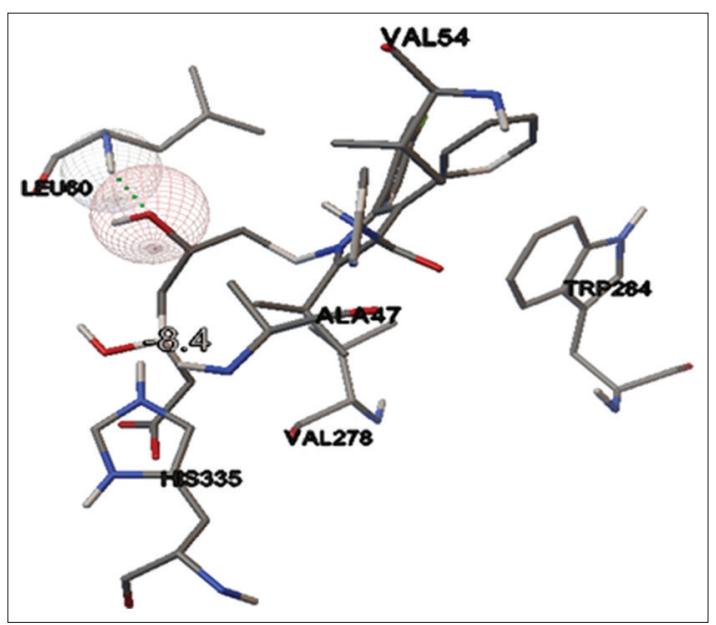

Fig. 7: Interaction of atorvastatin in the binding pocket of 3-hydroxy-3-methyl-glutayl-coenzyme A reductase

Structural modifications can be conducted to improve its binding affinity by keeping the pharmacophores [21].

In the present study, molecular docking simulations was conducted to confirm the inhibition of HMG Co-A reductase activity by predicting bonding between apigenin, apigetrin, simvastatin, and atorvastatin as ligands and HMG Co-A reductase enzyme as receptor (target molecule). It was done by predicting bonding conformation energy and molecule orientation in the active site for targeting receptors and modeling interactions between ligands and receptor analysis of the enzyme active HMG Co-A reductase was done by studying the interaction between apigenin, apigetrin as the substrate with the amino acid residues contained in the active site. HMG Co-A reductase as a target receptors obtained by downloading from Potein Data Bank (www.pdb.org) with PDB code: 4I6Y

In this method, the device used is ChemBioOffice Ultra 13.0 Suite (trial version), Ligand Explorer Viewer 4.1.0 (Online: http://www.pdb. 
org/pdb/explore/), Q-Site Finder (Online: http://www.modelling. leeds.ac.uk/qsitefinder/), YASARA 13.9.8, Autodock Vina, PyRx 0.8, 1.5.6 MGLTools (AutoDockTools-1.5.6), Chimera 1.8. In this method, ligands directed to various possible positions in the structure of target molecules. Docking method validation was done by redocking natural ligand receptor on the active side. Validation parameters docking methods were evaluated based on the value RMSD and declared valid if the value of RMSD $<2$ [22].

Based on the above results, the parameter calculations are fulfilling the criteria RMSD values which were lower than two. It showed that the calculation parameters met the validity of the method of docking. The entire docking parameters were used during validation and declared the method was valid then applied to the test compounds. The analysis was performed with energy of binding affinity (Ei), inhibition constants (Ki), hydrogen bonds, and interactions that occur between the ligand (apigenin, apigetrin, simvastatin, and atorvastatin) with an amino acid residue at the active site of receptors. The more negative of binding affinity showed good interaction between ligand and receptor.

\section{CONCLUSION}

Apigenin had higher binding affinity with HMG Co-A reductase compared to apigetrin. Apigenin could inhibit HMG Co-A reductase in silico better than apigetrin. Apigenin could be developed as anticholesterol.

\section{REFERENCES}

1. Guyton AC, Hall JE. Textbook of Medical Physiology. $11^{\text {th }}$ ed. Philadelphia, PA: Elsevier Saunders; 2006.

2. Ruela-de-Sousa RR, Fuhler GM, Blom N, Ferreira CV, Aoyama H, Peppelenbosch MP. Cytotoxicity of apigenin on leukemia cell lines: Implications for prevention and therapy. Cell Death Dis 2010;1:e19.

3. Kim B, Jung N, Lee S, Sohng JK, Jung HJ. Apigenin inhibits cancer stem cell-like phenotypes in human glioblastoma cells via suppression of c-met signaling. Phytother Res 2016;30(11):1833-40.

4. Si D, Wang Y, Zhou YH, Guo Y, Wang J, Zhou H, et al. Mechanism of CYP2C9 inhibition by flavones and flavonols. Drug Metab Dispos 2009;37(3):629-34.

5. Chakravarthi S, Thani PM, Yang DL, Husin LT, Lee N. Role of immunohistochemistry and apoptosis as investigative tools in assessing the prognosis of patients with prostate tumours. Exp Ther Med 2010;1(1):391-3.

6. Chong FW, Chakravarthi S, Nagaraja HS, Thanikachalam PM, Lee N. Expression of transforming growth factor-beta and determination of apoptotic index in histopathological sections for assessment of the effects of Apigenin (4', 5', 7'- trihydroxyflavone) on cyclosporine a induced renal damage. Malays J Pathol 2009;31(1):35-43.

7. Zbidah M, Lupescu A, Jilani K, Fajol A, Michael D, Qadri SM, et al. Apigenin-induced suicidal erythrocyte death. J Agric Food Chem 2012;60(1):533-8
8. Jung UJ, Cho YY, Choi MS. Apigenin ameliorates dyslipidemia, hepatic steatosis and insulin resistance by modulating metabolic and transcriptional profiles in the liver of high-fat diet-induced obese mice. Nutrients 2016;8(5). pii: E305.

9. Ali S, Srejith K. Multi targeted action of a plant derived flavonoids glucoside 'apigetrin' against important macromolecular targets in cancer an in silico validation. Int J Pharm Bio Sci 2014;5(3):478-90.

10. Lindgren V, Luskey KL, Russell DW, Francke U. Human genes involved in cholesterol metabolism: Chromosomal mapping of the loci for the low density lipoprotein receptor and 3-hydroxy-3-methylglutarylcoenzyme A reductase with cDNA probes. Proc Natl Acad Sci U S A 1985;82(24):8567-71.

11. Roitelman J, Olender EH, Bar-Nun S, Dunn WA Jr, Simoni RD. Immunological evidence for eight spans in the membrane domain of 3-hydroxy-3-methylglutaryl coenzyme A reductase: Implications for enzyme degradation in the endoplasmic reticulum. J Cell Biol 1992;117(5):959-73.

12. Iyan Sopyan, Achmad Fudholi, Muchtaridi, Ika Puspitasari. A simple effort to enhance solubility and dissolution rate of simvastatin using co-crystalization. Int J Pharm Pharm Sci 2016; 8(8): 342-6.

13. Jeyashanthini Nalaiya, Sreenivasa Rao Sagineedu, Rajakumari Rajasingam, Zawahil Kassim, Mallikarjuna Rao Pichika. A stability indicating RP-HPLC method for simultaneous determination of simvastatin and niacin in a combined dosage form. Int J Pharm Pharm Sci 2015; 7(2): 262-8.

14. Cechinel-Filho V. Plant Bioactives and Drug Discovery: Principles, Practice and Perspective. Hoboken, NJ, Canada: John Wiley \& Sons; 2012. p. 104

15. Miean KH, Mohamed S. Flavonoid (myricetin, quercetin, kaempferol, luteolin, and apigenin) content of edible tropical plants. J Agric Food Chem 2001;49(6):3106-12.

16. Jeong GS, Lee SH, Jeong SN, Kim YC, Kim EC. Anti-inflammatory effects of apigenin on nicotine- and lipopolysaccharide-stimulated human periodontal ligament cells via heme oxygenase-1. Int Immunopharmacol 2009;9(12):1374-80.

17. Wei H, Tye L, Bresnick E, Birt DF. Inhibitory effect of apigenin, a plant flavonoid, on epidermal ornithine decarboxylase and skin tumor promotion in mice. Cancer Res 1990;50(3):499-502.

18. Brown MS, Goldstein JL. The SREBP pathway: Regulation of cholesterol metabolism by proteolysis of a membrane-bound transcription factor. Cell 1997;89(3):331-40.

19. WHO. 19th WHO Model List of Essential Medicines. [Last retrieved on 2015 May 10].

20. Marcou G, Rognan D. Optimizing fragment and scaffold docking by use of molecular interaction fingerprints. J Chem Inf Model 2007; $47: 195-207$.

21. Steussy CN, Critchelow CJ, Schmidt T, Min JK, Wrensford LV, Burgner JW $2^{\text {nd }}$, et al. A novel role for coenzyme A during hydride transfer in 3-hydroxy-3-methylglutaryl-coenzyme A reductase. Biochemistry 2013;52:5195-205.

22. Trott O, Olson AJ. Autodock Vina: Improving the speed and accuracy of docking with a new scoring function, efficient optimization, and multithreading. J Comput Chem 2010;31(2):455-61. 